\title{
Physiological and Molecular Effects of in vivo and ex vivo Mild Skin Barrier Disruption
}

\author{
Eva K.B. Pfannes ${ }^{a}$ Lina Weiss ${ }^{a}$ Sabrina Hadam ${ }^{a}$ Jessica Gonnet ${ }^{b}$ \\ Béhazine Combardière $^{b} \quad$ Ulrike Blume-Peytavi ${ }^{a} \quad$ Annika Vogt $^{a, b}$ \\ ${ }^{a}$ Charité - Universitätsmedizin Berlin, corporate member of Freie Universität Berlin, Humboldt-Universität zu Berlin, \\ and Berlin Institute of Health, Clinical Research Center for Hair and Skin Science, Department of Dermatology \\ and Allergy, Berlin, Germany; ${ }^{b}$ Centre d'Immunologie et des Maladies Infectieuses - Paris (Cimi-Paris), Sorbonne \\ Universités UPMC, Université Paris 06 UMRS CR7, Inserm U1135, CNRS ERL 8255, Paris, France
}

\section{Keywords}

Skin barrier · Cyanoacrylate skin surface stripping · Transcutaneous vaccination · Particle penetration · Tape stripping

\begin{abstract}
The success of topically applied treatments on skin relies on the efficacy of skin penetration. In order to increase particle or product penetration, mild skin barrier disruption methods can be used. We previously described cyanoacrylate skin surface stripping as an efficient method to open hair follicles, enhance particle penetration, and activate Langerhans cells. We conducted ex vivo and in vivo measurements on human skin to characterize the biological effect and quantify barrier disruption-related inflammation on a molecular level. Despite the known immunostimulatory effects, this barrier disruption and hair follicle opening method was well accepted and did not result in lasting changes of skin physiological parameters, cytokine production, or clinical side effects. Only in ex vivo human skin did we find a discrete increase in IP-10, TGF- $\beta$, IL-8, and GM-CSF mRNA. The data underline the safety profile of this method and demonstrate that the pro-
\end{abstract}

\section{KARGER}

(c) 2018 S. Karger AG, Basel

E-Mail karger@karger.com

www.karger.com/spp cedure per se does not cause substantial inflammation or skin damage, which is also of interest when applied to noninvasive sampling of biomarkers in clinical trials.

(c) 2018 S. Karger AG, Basel

\section{Introduction}

The skin permeation of drugs depends on the site of application and is strongly correlated with the hair follicle density [1]. The hair follicle is a reservoir for penetrated substances [2], and thus procedures to enhance this penetration pathway are valuable tools in dermatopharmaceutic research. Differential stripping methods have been widely used for investigations into the penetration of topically applied substances $[3,4]$ and have even been proposed as methods to enhance penetration, e.g., in transcutaneous vaccination. With regard to hair follicle penetration cyanoacrylate skin surface stripping (CSSS), a variation of stripping using cyanoacrylate-based resins, is of special interest. Because the liquid resin flows into hair follicle

Eva K.B. Pfannes and Lina Weiss contributed equally to this work. 
openings, it has been successfully applied for recovery of skin surface material and hair follicle material [5-7], as well as proposed as a penetration enhancement method [8-10]. For both types of application, it is important to understand the biological effects induced by the procedure not only in ex vivo experiments, but also in in vivo settings.

Quantification of protein extracted from tapes is frequently used for the investigation of interleukins and cytokines in healthy and diseased skin and scalp $[6,11-14]$. Because CSSS removes not only skin surface material, but approximately $30 \%$ of the stratum corneum [10], biomarkers extracted from such material may yield more relevant information compared to conventional adhesive tape strips. Several clinical studies suggest good tolerability and safety, but possible biological effects or the extent of the additional inflammatory stimulus caused by the procedure have not been investigated. Such information is especially important when CSSS is integrated into clinical trial protocols. It is known that even conventional adhesive tape strips induce substantial changes in gene expression. E.g., on a molecular level repetitive tape stripping induced gene expression of different cytokines and chemokines, including TNF- $\alpha$, IL- $1 \alpha$, and IL- $1 \beta[15,16]$. Defensins were found to be increased after repetitive tape stripping in a murine model [17], and epidermal mRNA expression of TNF- $\alpha$, HSP-70, IL-33, and IL-8/CXCL8 were increased in human biopsy samples [18].

Such biological effects become even more important with regard to the recently proposed use of CSSS as a method for penetration enhancement and immune activation in transcutaneous immunization patches $[9,19]$. CSSS is highly interesting for transcutaneous immunization as it targets the hair follicles as vaccine reservoir and immune organ. Data obtained from an ex vivo human skin model showed that the opening of hair follicles for penetration, the mild barrier disruption, and the subsequent pre-activation of dendritic cells were key to success of this vaccine delivery approach [20] and, besides being non-invasive, contributed to the observed induction of CD8 immune responses [19].

As the relevance of biomarkers increases in skin penetration research and also with regard to cell-targeting strategies, inflammatory effects caused by the procedure itself are important aspects of interest to investigate the benefit of CSSS. It is essential to evaluate the extent and the quality of procedure-related biological effects.

Herein, we show ex vivo and in vivo results on the effects of conventional adhesive tape stripping or CSSS on skin physiological parameters, cytokine and chemokine expression in human skin.

\section{Materials and Methods}

\section{In vivo Experiments}

Explorative Clinical Study

An explorative clinical non-drug study was performed on 12 volunteers ( 5 male and 7 female, average age 32.2 years, phototypes between II and III according to the Fitzpatrick classification). Ethic approval EA1/132/13 was obtained from the ethics committee, Charité - Universitätsmedizin Berlin. The clinical study was conducted at the Clinical Research Center for Hair and Skin Science, Department of Dermatology, Charité - University Medicine Berlin, according to the ethical rules stated in the Declaration of Helsinki. The aim of the study was to assess changes in skin barrier function and integrity after CSSS compared to conventional adhesive tape stripping. Each volunteer was treated with 1 CSSS on 1 upper arm (below the deltoid region) and 1 adhesive tape on the other upper arm. Left or right sides were assigned to the procedures by randomization. Both CSSS and tape stripping remained attached to the skin for $20 \mathrm{~min}$. For a visual graphic of study organization, please refer to online supplementary Figure 1 (for all online suppl.material, seewww.karger.com/doi/10.1159/000484443).

\section{In vivo CSSS and Tape Stripping}

Two investigational sites of $4 \mathrm{~cm} \times 4 \mathrm{~cm}$, one on the upper left arm and another on the upper right arm, were each marked with a permanent skin marker (skin marker H7003 Falc). Subjects had to acclimatize in the measurement rooms for at least $30 \mathrm{~min}$ with the upper arms uncovered. Measurement rooms were temperature and humidity controlled $\left(21 \pm 2{ }^{\circ} \mathrm{C}\right.$ and $50 \pm 10 \%$ humidity). Areas were marked and dry-shaved before the acclimatization time. A dry razor was used to shave the investigational test areas (Disposable razor, Art. No. 183 H, Wilkinson Sword GmbH, Germany). For the singlestep CSSS procedure, $180 \mathrm{mg}$ of cyanoacrylate glue (9 drops, UHU Sekunden Alleskleber geruchsfrei Easy, UHU GmbH, Buehl/Baden, Germany) was spread onto the skin surface with a glass slide. Adhesive tape $\left(6 \times 5 \mathrm{~cm}\right.$, Art. No. 57176-00000, $66 \mathrm{~m} \times 50 \mathrm{~mm}$, tesa ${ }^{\circledR}$ Beiersdorf, Beiersdorf, Germany) was cut to fit the test area size, then applied to the skin and massaged with a roll rubber $(4.5 \mathrm{~cm}$ diameter, Lux series, OBI, Germany) 5 times back and forth to improve adhesion. For the single tape stripping, the procedure was identical, but no cyanoacrylate glue was applied. Both CSSS and tape stripping materials were removed after $20 \mathrm{~min}$ attached to the skin.

\section{Measurement of Cytokines in Blood after CSSS}

For an additional 5 subjects, blood samples were collected after 1 single $16-\mathrm{cm}^{2}$ CSSS. IL-12p70, IL-10, IL-6, TNF- $\alpha$, IL- $1 \beta$, and IL-8 were measured using the Human Inflammation Kit BD Cytometric Bead Array (BD Biosciences, San Diego, CA, USA) according to manufacturer instructions.

In vivo Skin Physiology Measurements and Skin Surface Protein Harvesting and Protein Extraction

Measurements were performed using a Multi Probe Adapter MPA (Courage-Khazaka, Cologne, Germany). For details of skin physiology measurements and an overview of the methods, please refer to the supplementary material (see online suppl. Fig. 1). Stratum corneum material was collected at different time points to quantify cytokines using Sebutape (S100 Sebutape patches, CuDerm Corporation, Dallas, TX, USA). Details on protein harvesting and extraction can be found in the supplementary material. 
Determination of Total Protein and ELISA

After protein extraction, total protein of samples was determined using the Micro BCA protein assay kit (No. 23235, Thermo Fisher Scientific Inc., Waltham, MA, USA) using bovine serum albumin as a standard. ELISA assays from Sebutape-extracted protein material for IL-1 $\alpha$, IL-1 $\beta$, IL-1RA (DY200, DY201, and DY280, respectively, all from R \& D Systems Inc.), for IL-6 (CytoSet ${ }^{\mathrm{TM}}$ No. CHC1263, Invitrogen Corporation, Camarillo, CA, USA), and IL-8 (CytoSet ${ }^{\mathrm{TM}}$ No. CHC1303, Invitrogen Corporation, Camarillo, USA) were performed according to instructions provided by the kits.

Acceptability and Evaluation of Pain

Acceptability and pain perceived during the procedures were assessed using questionnaires and a visual analogue scale for pain.

\section{Ex vivo Experiments}

Ex vivo Skin Samples

Fresh skin samples were obtained from 7 healthy volunteers undergoing plastic surgery of the breast $(n=1)$ or the abdomen $(n=6)$. All skin samples were taken after informed consent approval by the Institutional Ethics Committee of the Charité Universitätsmedizin Berlin, according to the ethical rules stated in the Declaration of Helsinki. All donors $(n=7)$ were female between 32 and 63 years of age (average age 45.8 ) and had phototypes between I and III according to the Fitzpatrick classification. After skin preparation (please refer to suppl. material), treatments and measurements were conducted.

Ex vivo Skin Physiology Measurements, Treatments, and Measurements

Skin physiology measurements were performed using a Multi Probe Adapter MPA (Courage-Khazaka, Cologne, Germany). For details, see supplementary materials.

Physical Barrier Disruption. Three fields of $1 \mathrm{~cm}^{2}$ each were marked on the skin. The fields were $1 \mathrm{~cm}$ apart from each other. One of them was left without treatment, on another a single tape stripping was performed using adhesive tape (TESA Beiersdorf, catalog No. 57176-00000, $66 \mathrm{~m} \times 50 \mathrm{~mm}$ ). To ensure standardized adhesion a rubber roll was used to evenly press the tape onto the skin surface. The tape remained on the skin for $20 \mathrm{~min}$. On a third field mini-zone CSSS was performed as previously described [6, 20] adding 1 drop of cyanoacrylate (UHU Sekunden Alleskleber geruchsfrei EASY, catalog No. 46130, UHU GmbH \& Co. KG, Germany), corresponding to $20 \mathrm{mg}$, before pressing on the adhesive tape.

Sequence of Skin Measurements. Skin physiology measurements were performed prior to skin barrier disruption and after an incubation period of $24 \mathrm{~h}$ in a wet chamber at $37^{\circ} \mathrm{C}$. Skin physiology parameters were measured as described in the supplementary material. Skin surface material was collected using Sebutape (Sebutape $^{\circledR}$, CuDerm Corp., USA) and stored in 15-mL tubes (Fal$\operatorname{con}^{\circledR}$, Corning Science México S.A. de C.V., México) using a forceps and frozen at $-80^{\circ} \mathrm{C}$.

Protein Extraction from Diagnostic Sebutape and CSSS

To quantify different cytokines and chemokines, protein material was extracted from the diagnostic strips (Sebutape and minizone CSSS). The mini-zone CSSS strips were diluted according to Pfannes et al. [6]. For a detailed description, please refer to the online supplementary material.

Mild Skin Barrier Disruption

\section{Statistical Analysis}

For the statistical analysis, 1-tailed paired Wilcoxon tests were performed using GraphPad Prism 5.0 (GraphPad Software Inc., La Jolla, CA, USA). For skin physiology measurements different treatment methods were compared, and within the treatments groups changes over time were evaluated. For IL- $1 \alpha$ analysis different treatment methods were compared.

\section{RNA Extraction and $q R T-P C R$}

For details on skin samples and skin preparation, please refer to the supplementary material. An RNeasy Mini Kit was used (Qiagen $\mathrm{GmbH}$, Germany) for cell suspensions of up to $7 \times 10^{6}$ cells. The extracted RNA amount was quantified using a Nanodrop (Thermo Scientific, Wilmington, NC, USA). The RNAs extracted from the epidermis of 6 samples of each donor were diluted to the same concentration, and a SuperScript ${ }^{\circledR}$ VILO cDNA Synthesis Kit (Life Technologies) was used for reverse transcription into cDNA. Afterwards the cDNA was diluted to a concentration of $1 \mathrm{ng} / \mu \mathrm{L}$, and $5 \mathrm{ng}$ ( $5 \mathrm{ng} / \mu \mathrm{L}$ to $20 \mathrm{ng}$ for CXCL9, GM-CSF, IFN- $\gamma$, IL-6, chemerin) were used for quantitative PCR using SYBR ${ }^{\circledR}$ Green PCR Master Mix (Life Technologies, Thermo Scientific, USA) and QuantiTect Primer Assays (Qiagen GmbH, Germany) for 17 genes (IL-1 $\alpha$, IL-1 $\beta$, TNF- $\alpha$, CCL22, CXCL9, IL-8, IL-16, chemerin, CX3CL1, IP-10, GM-CSF, TGF- $\beta$, CCR7, IL-6, IL-1RA, CCL2, and IFN- $\beta$ ) tested.

\section{Analysis}

Semiquantitative quantification of RNA sequences was done by comparing $\Delta \mathrm{C}_{\mathrm{T}}$ values, i.e., the gene-specific $\mathrm{C}_{\mathrm{T}}$ value was normalized to the mean $\mathrm{C}_{\mathrm{T}}$ value of the housekeeping genes GADPH and $\beta$-actin for each sample. A normalization sample was used on every plate to account for interplate variability. For the statistical analysis, 1-tailed paired Wilcoxon tests were performed using Prism 5.0. Significance was recognized for samples where the difference between treatments was larger than the standard deviation of the normalization samples.

\section{Results}

Changes on skin barrier function can be measured using transepidermal water loss (TEWL), which increases as a function of tape strip number [21]. Although limited by high interindividual variations, TEWL is a valuable tool for the investigation of skin barrier turnover in response to disruption methods. The degree of barrier disruption is influenced by the character of the tape and the method of application [22]. A marked increase in TEWL values was observed for approximately $5 \mathrm{~min}$ after CSSS and adhesive tape procedures in vivo (Fig. 1a). Compared to tape stripping, a return to baseline was slightly delayed in CSSS-treated areas. Stratum corneum hydration slightly increased over time after both interventions (Fig. 1b). The skin surface $\mathrm{pH}$ decreased after CSSS (Fig. 1c), which is in accordance with the larger amount of stratum corneum removed compared to single tape stripping [20]. 


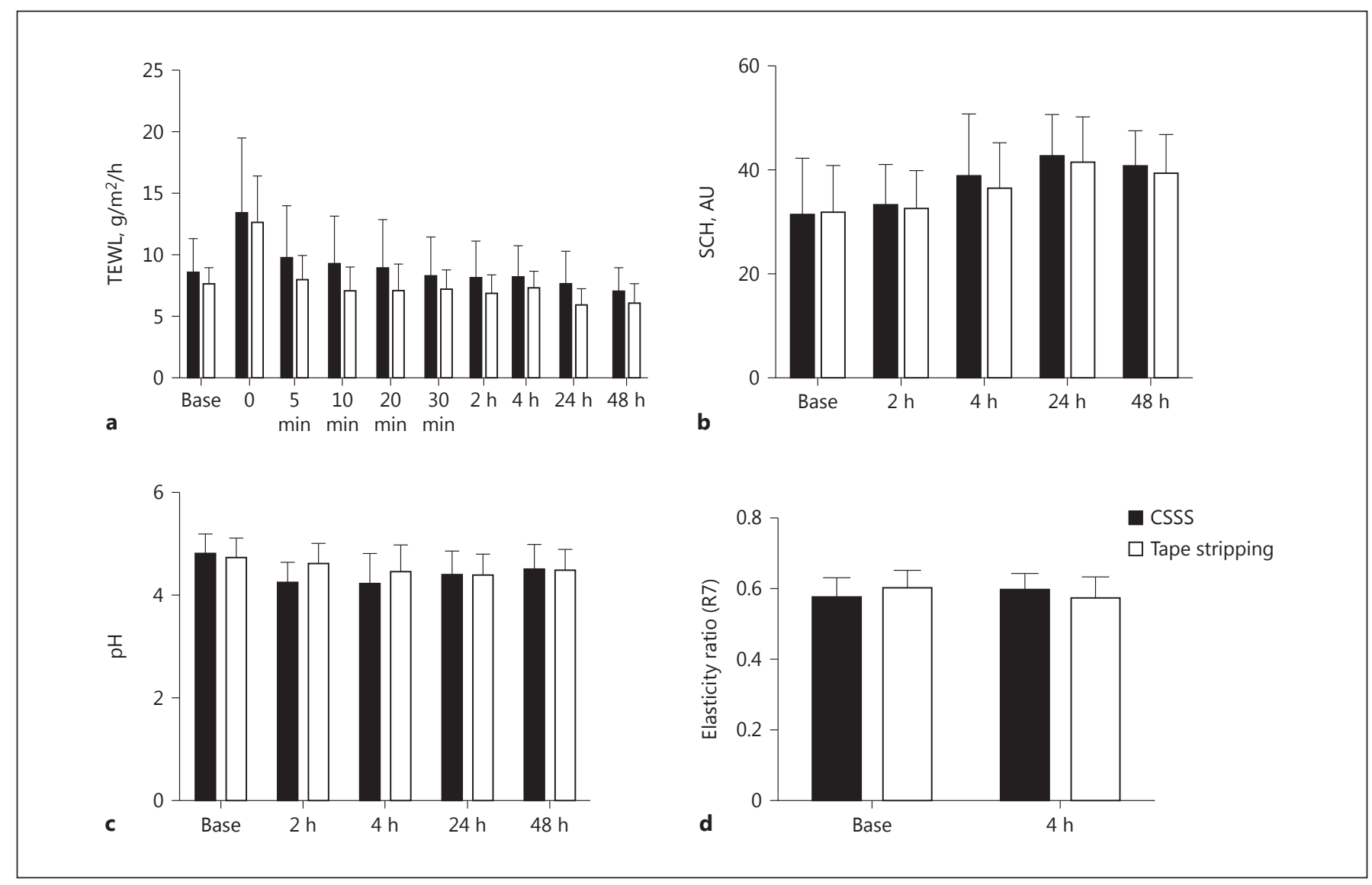

Fig. 1. Skin physiology measurements after CSSS compared to a single adhesive tape stripping procedure in vivo. An increase in TEWL was observed for both CSSS and tape directly after the procedure (a), with a quick recovery after $5 \mathrm{~min}$. A small recovery delay was observed for the CSSS test areas. Stratum corneum hy-

dration ( $\mathrm{SCH}$ ) equally increased for both procedures (b), whereas pH strongly decreased after CSSS (c). No changes in skin elasticity (d) were observed. $n=12$. Mean values are depicted, error bars represent standard deviation. "Base" stands for baseline and represents values before procedures (before skin barrier disruption).

No changes in skin elasticity were observed (Fig. 1d). Levels of IL-1 $\alpha$, IL- 6 , and IL- 8 collected by Sebutapes were determined before (baseline) and after (directly after, after $2 \mathrm{~h}$, after $4 \mathrm{~h}$, after $24 \mathrm{~h}$, and after $48 \mathrm{~h}$ ) both treatments in vivo. The amount of interleukin per Sebutape was normalized to the amount of total soluble protein in the sample. A slight increase in IL-1 $\alpha$, a constitutively produced mediator, and a decrease in IL-1RA (receptor antagonist) (Fig. 2a and b, respectively) were observed. This effect was more pronounced after single tape stripping, probably because the preformed reservoir of IL-1 $\alpha$ in the upper stratum corneum compartment was removed by CSSS. No new induction of IL-6 and IL-8, 2 inflammatory markers known to be produced in response to stress stimuli and marked skin barrier disruption, was observed in vivo (data not shown).

Volunteers indicated in the questionnaires that they would readily choose CSSS-based transcutaneous vaccination as the vaccination method if available (11/12 strongly agree), that they would prefer it over intramuscular injections given that the effects were equal (10/12 strongly agree) and that they would consider CSSS-based vaccinations if that would allow to get protected against diseases for which no intramuscular vaccination exists (11/12 strongly agree). Pain assessment by visual analogue scale revealed that pain perceived after intramuscular injection was rated markedly higher than CSSS and regular adhesive tape stripping (see online suppl. Fig. 2).

No changes in interleukins measured in blood after 1 single CSSS were observed. For IL-12p70 and TNF- $\alpha$, results were negative. Results for IL-10, IL-6, IL-1 $\beta$, and IL-8 are shown in Figure 3. 


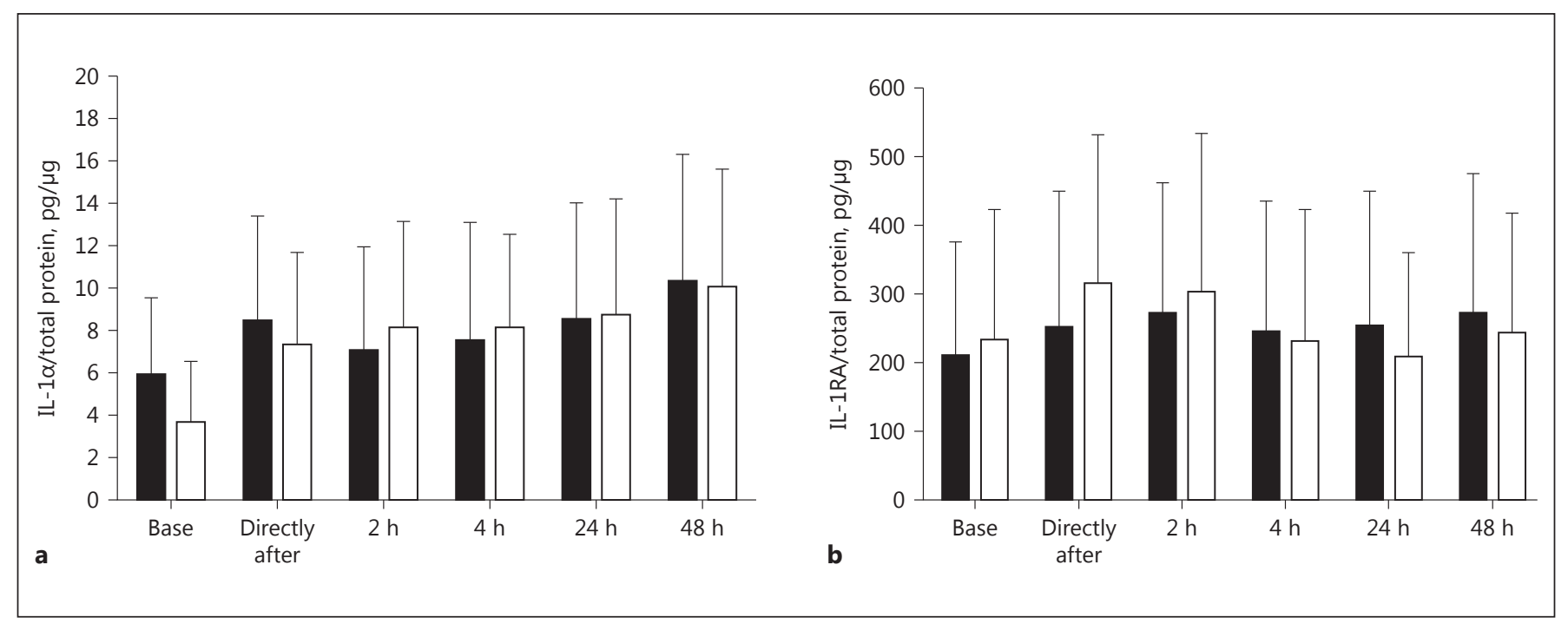

Fig. 2. a IL-1 a content (normalized by total protein content) over time before and after cyanoacrylate skin surface stripping (CSSS) or single adhesive tape stripping (TS) in vivo. b IL-1RA content over time. $n=10$. Mean values are depicted, error bars represent standard deviation. In black, results for CSSS and in grey, results for tape stripping are shown. "Base" stands for baseline and represents values before procedures (before skin barrier disruption).
We further investigated skin physiology in ex vivo skin samples at different time points (Fig. 4a). No significant differences were observed in TEWL before and after $24 \mathrm{~h}$ barrier disruption, similar to the in vivo result after $24 \mathrm{~h}$. A significant increase in stratum corneum hydration was observed in CSSS-treated skin $24 \mathrm{~h}$ after disruption. Similar to in vivo measurements a lower skin surface $\mathrm{pH}$ was noticeable after $24 \mathrm{~h}$ in untreated as well as in disrupted skin, reaching a level of significance in CSSS. Similar to our in vivo results, levels of IL- $1 \alpha$, IL-1 $\beta$, and IL-1RA were analysed from Sebutapes $24 \mathrm{~h}$ after the disruption procedures in ex vivo skin and normalized to overall protein content (Fig. 4b). For the ex vivo samples the levels of IL- $1 \beta$ and IL-1RA were lower than the detection minimum of the used kits. For IL-1 $\alpha$, similar to our in vivo measurements, the detected amount was highest after single tape stripping. There was no noticeable difference between CSSS-treated and non-disrupted skin.

For an additional analysis of the epidermal cytokine and chemokine milieu, qRT-PCR for a wide range of inflammatory mediators was performed on RNA extracts from ex vivo human skin samples. A preliminary selection of molecules of interest was performed according to results from a chemokine protein array (data not published). Immunologically relevant molecules were selected and in total 17 different genes were tested: IL- $1 \alpha$, IL-1 $\beta$, TNF- $\alpha$, CCL22, CXCL9, IL-8, IL-16, chemerin,
CX3CL1, IP-10, GM-CSF, TGF- $\beta$, CCR7, IL-6, IL-1RA, CCL2, and IFN- $\beta$. Figure 5 shows a selection of molecules we investigated in our experiments. $\Delta \mathrm{C}_{\mathrm{T}}$ values were compared between CSSS-treated and non-treated skin. While IL- $1 \alpha$ and IL-1 $\beta$ did not show major differences in gene expressions, IL-1RA indicated a lower gene expression after CSSS. This is in agreement with the literature since an increased expression would be expected at an earlier time point and an increased IL-1/IL-1RA ratio as an indicator for inflammation [23-26]. A significantly increased expression was observed for IP-10 (16 h) and TGF- $\beta$ ( $4 \mathrm{~h}$ ) compared to untreated skin. Similarly, increased IL-8 and GM-CSF expression levels were found after 4 and $16 \mathrm{~h}$ of the CSSS procedure (Fig. 5). An increase at time point $4 \mathrm{~h}$ and a subsequent decrease at time point $24 \mathrm{~h}$ were observed for IL- 6 after CSSS.

\section{Discussion}

In vivo and ex vivo skin physiology measurements are comparable with each other. Mean TEWL baseline values are higher in vivo than ex vivo. Stratum corneum hydration showed a slight increase for both CSSS and tape stripping in vivo. In the ex vivo experiments this result is statistically significant after CSSS probably as a result of controlled temperature and humidity during the 


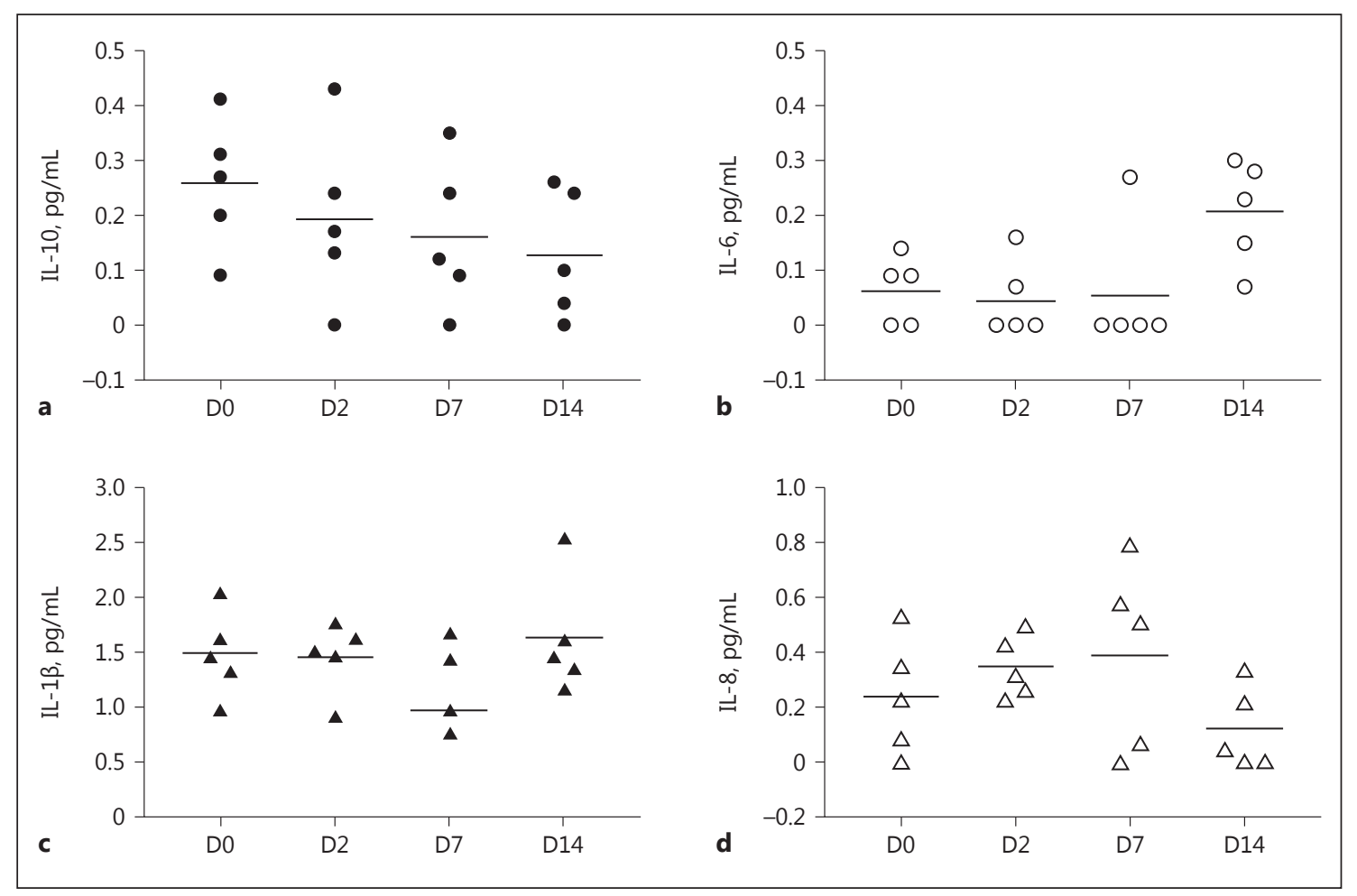

Fig. 3. Interleukin measured in blood after 1 single CSSS $\left(16 \mathrm{~cm}^{2}\right)$. a Results for IL-10. b For IL-6. c For IL-1 1 . d For IL-8. Each spot represents a single subject $(n=5)$. Mean values are shown as a black line. Results are below the threshold of positivity of the assay; thus, CSSS does not induce prolonged alteration of interleukins in blood. Blood samples were taken om day 0 (D0), day 2 (D2), day 7 (D7), and day 14 (D14).

incubation. $\mathrm{pH}$ values do not significantly change in vivo or ex vivo.

Despite CSSS being the more vigorous procedure, IL$1 \alpha$ levels were higher after single adhesive tape stripping both in vivo and ex vivo (Fig. 2, 4). This is in accordance with the storage function of stratum corneum for preformed IL- $1 \alpha$ especially in superficial compartments. The origin of this cytokine was already reported to derive from an intracellular pool of damaged corneocytes and membrane-bound IL-1a [27]. This reservoir is substantially reduced by CSSS compared to adhesive tape stripping $[23,28]$.

We observed that the CSSS procedure was well tolerated and well accepted by all volunteers, and found no indication for marked inflammation within the first $48 \mathrm{~h}$ after the procedure. Skin barrier disruption after CSSS appeared to be only slightly higher compared to regular adhesive tape recovering fast after initial rise in TEWL. Clearly the study was designed as an explorative study in a small cohort $(n=12)$, and inclusion of more individuals may provide more substantial data. As for topical ap- plications and transcutaneous drug delivery [10, 29], however, there seems to be an optimal time window for application directly after removal of the tape, i.e., both steps should be performed shortly after each other [10, 29]. Late-stage (after 7 days and after 14 days) in vivo measurement of interleukin in blood after a single CSSS did not show significant changes. Those results were below the threshold of positivity of the assay provided by the manufacturer. They demonstrate that CSSS per se does not cause measurable systemic inflammatory responses. Increases in IL-8 observed in transcutaneous vaccinated individuals were most likely the result of the combined use of CSSS and the topically applied vaccine [30]. In accordance with our in vivo data, our ex vivo results showed only minor changes in skin physiology measurements. Our findings are in line with safety and acceptability of ongoing clinical trials where local side effects of the procedure mostly resulted in transient erythema.

Despite finding small differences in interleukin secretion in skin surface samples at specific time points in vivo, 


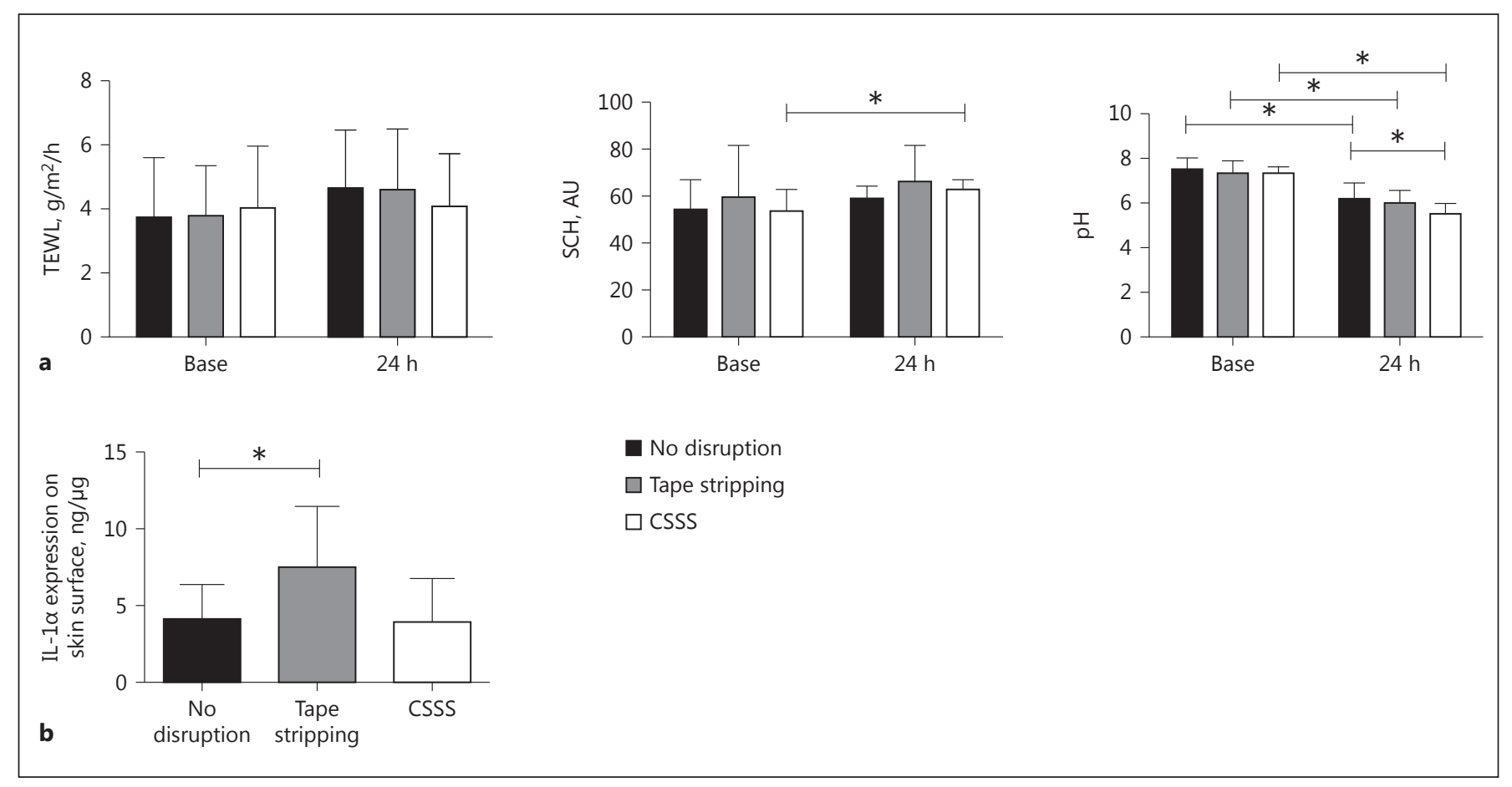

Fig. 4. Skin physiology and cytokine measurements after 1 CSSS compared to a single tape stripping and untreated skin in ex vivo human skin. a TEWL measurements at different time points show no major differences between treatment methods. A significant decrease in $\mathrm{pH}$ at $24 \mathrm{~h}$ is observed after CSSS compared to nondisrupted skin. $\mathrm{SCH}$, stratum corneum hydration (capacitance). b IL-1a expression on the skin surface $24 \mathrm{~h}$ after treatment. A sig- nificant increase in the amount of IL-1a on tape-stripped skin compared to non-disrupted skin is observed. No noticeable changes in IL-1a expression after CSSS could be detected. Mean values and standard deviations are depicted. * $p<0.05$. "Base" stands for baseline and represents values before procedures (before skin barrier disruption). we know from previous studies that CSSS promotes activation of Langerhans cells (LCs) and cellular uptake [10]. Thus qRT-PCR allowed for additional analysis of the epidermal cytokine and chemokine milieu in ex vivo samples. Although no changes were observed in the IL-1 $\alpha$, IL-1 $\beta$ and IL-1RA levels after CSSS either in skin surface analysis or in qRT-PCR, an increase of expression would be expected at an earlier time point $[16,23-25,28]$. This is also valid for IL-6 expression, a cytokine that plays a role in repair mechanisms and dendritic cell activation, and with highest protein levels generally detected between 2 and $7 \mathrm{~h}$ after barrier disruption, supported by our ex vivo results of higher expression $4 \mathrm{~h}$ after CSSS treatment $[15,16,23,24,31]$. For IL-8, an indicator for severity of inflammation and attractant of neutrophils, our qRT-PCR results indicate a barrier reconstitution after 24 $\mathrm{h}$, which is coherent with our findings in skin physiology measurements for ex vivo probes [32, 33]. IL-8 is a chemoattractant for neutrophils that also play a role in linking neutrophils to the adaptive immunity [34]. An in- crease is normally seen shortly after barrier perturbation as a sign of inflammation $[15,16,35]$. Interestingly, our early trials using CSSS for transcutaneous immunization also pointed towards systemic cytokine increase after influenza transcutaneous vaccination, e.g., IL-8 was elevated in transcutaneous vaccinated individuals [19]. An increase in expression of GM-CSF, a pro-inflammatory cytokine that enhances neutrophil and monocyte function, was observed indicating a role in early immune response $[23,24,34,35]$. The ex vivo qRT-PCR measurements showed a change in gene expression for IP-10 and TGF- $\beta$, both modulators that contribute to the induction of Langerhans cells and dendritic cell maturation as well as the positioning of CXCR3-positive T cells in the skin [16, 23, $24,36]$. IP-10 together with IFN has already been described as an important cytokine induced by barrier disruption methods like tape stripping $[37,38]$. A 2-phased reaction of skin to barrier disruption has been described [39]. Some authors found a Th2- and eosinophil-focused response after tape stripping as well as increased IgG tit- 


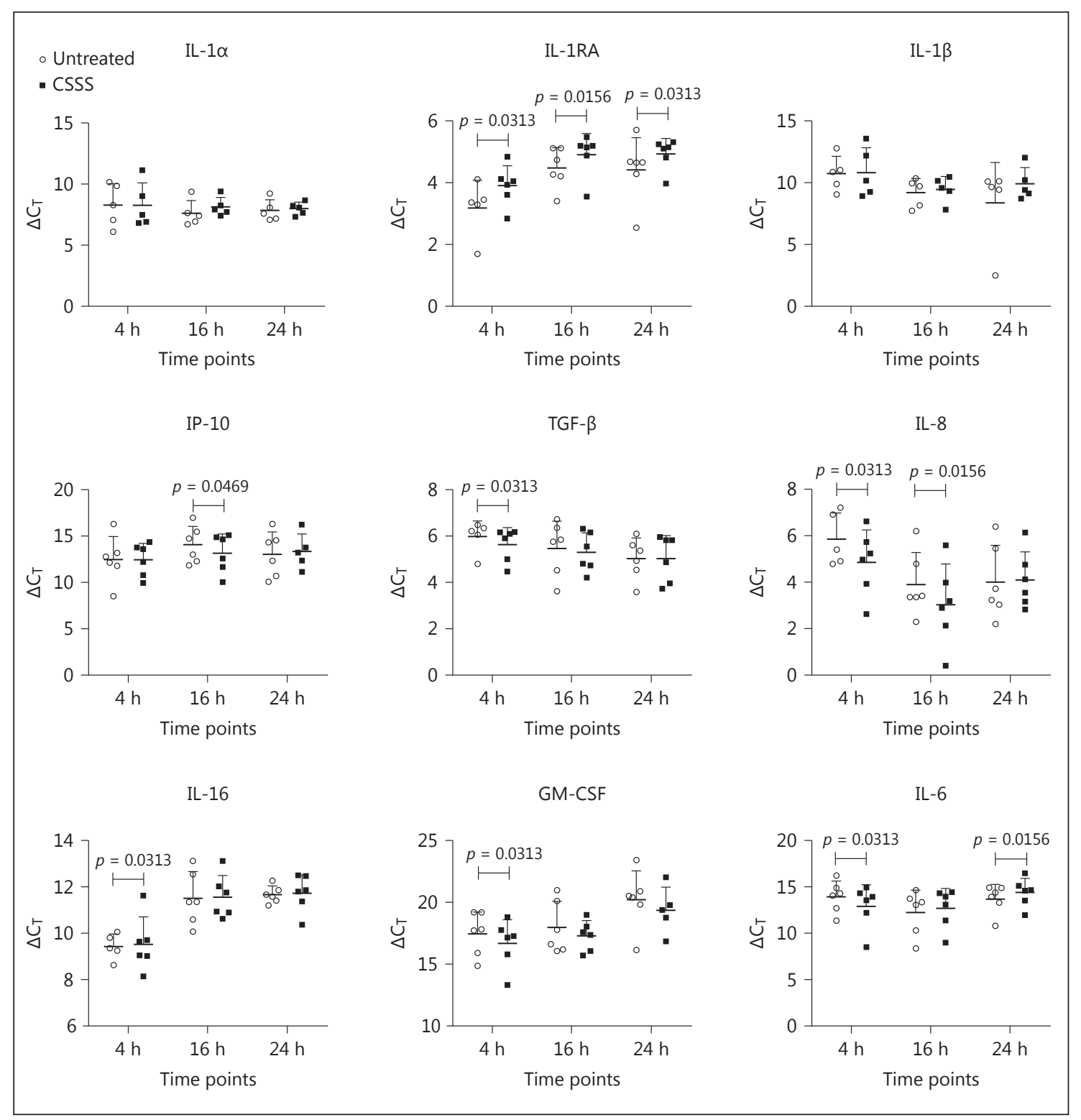

Fig. 5. $\Delta \mathrm{C}_{\mathrm{T}}$ values of a selection of molecules analysed by $\mathrm{qRT}$-PCR in epidermal cell suspensions not treated or after CSSS at 3 different time points $(4,16$, and $24 \mathrm{~h})$ are shown. A normalization sample was used on every plate to account for interpolate variability. For the statistical analysis, 1-tailed paired Wilcoxon tests were performed. Significant differences were seen for IL-1RA, IP-10, TGF- $\beta$, IL-8, IL-16, and IL-6 at different time points.

ters of anti-ovalbumin after application of a correspondent vaccine when applied on tape-stripped skin $[38,40$, 41]. In this context different vaccine formulations might be worth comparing since other findings suggest higher antigen amount distribution to the hair follicle when the compound is encapsulated in nanoparticles [42]. We therefore conclude that while CSSS does not provoke massive barrier disruption it still presents an immuno- logical stimulus. This information in combination with our other experimental ex vivo studies, where an increased follicular penetration was detected accompanied by the creation of a particle reservoir [20], shows the potential this method bears for transcutaneous vaccination and delivery strategies inducing only slight inflammation. 
The hair follicle is filled with sebum and scaled stratum corneum [2], and CSSS arises as a method that is able to open the hair follicle and prepare it for drug delivery. The removed protein material might be additionally quantified and analysed for inflammatory markers [6]. Thus, this mild barrier disruption method has the potential to become a simple, well-accepted diagnostic and penetration enhancement tool for dermatological research.

\section{Acknowledgement}

The authors kindly thank the European Commission for their financial support. Experiments were supported by a CUTHIVAC project grant from the European Commission (FP7).

\section{Statement of Ethics}

All skin samples were taken after informed consent approval by the Institutional Ethics Committee of the Charité - Universitätsmedizin Berlin, according to the ethical rules stated in the Declaration of Helsinki . For the in vivo measurements ethical approval EA1/132/13 was obtained from the ethics committee, Charité Universitätsmedizin Berlin. The clinical study was conducted at the Clinical Research Center for Hair and Skin Science, Department of Dermatology, Charité - University Medicine Berlin, according to the ethical rules stated in the Declaration of Helsinki.

\section{Disclosure Statement}

The authors report no conflict of interest.

\section{References}

1 Ogiso T, Shiraki T, Okajima K, Tanino T, Iwaki M, Wada T: Transfollicular drug delivery: penetration of drugs through human scalp skin and comparison of penetration between scalp and abdominal skins in vitro. J Drug Target 2002;10:369-378.

2 Vogt A, Hadam S, Heiderhoff M, Audring H, Lademann J, Sterry W, Blume-Peytavi U: Morphometry of human terminal and vellus hair follicles. Exp Dermatol 2007;16:946-950.

3 Ossadnik M, Czaika V, Teichmann A, Sterry W, Tietz HJ, Lademann J, Koch S: Differential stripping: introduction of a method to show the penetration of topically applied antifungal substances into the hair follicles. Mycoses 2007;50:457-462.

4 Thielitz A, Helmdach M, Ropke EM, Gollnick $\mathrm{H}$ : Lipid analysis of follicular casts from cyanoacrylate strips as a new method for studying therapeutic effects of antiacne agents. Br J Dermatol 2001;145:19-27.

5 Schwartz JR, Shah R, Krigbaum H, Sacha J, Vogt A, Blume-Peytavi U: New insights on dandruff/seborrhoeic dermatitis: the role of the scalp follicular infundibulum in effective treatment strategies. Br J Dermatol 2011;2: $18-23$.

6 Pfannes EK, Hadam S, Doge N, Fimmel S, Blume-Peytavi U, Vogt A: Mini-zone cyanoacrylate skin surface stripping: a new method for non-invasive sampling of scalp material. Exp Dermatol 2016;25:555-556.

7 Vogt A, Pfannes EKB, Fimmel S, Hadam S, Andruck A, Kottner J, Blume-Peytavi U: Infundibular protein and RNA microarray analyses from affected and clinically non-affected scalp in male androgenetic alopecia patients. Exp Dermatol 2017;26:518-521.
8 Mahe B, Vogt A, Liard C, Duffy D, Abadie V, Bonduelle O, Boissonnas A, Sterry W, Verrier B, Blume-Peytavi U, Combadiere B: Nanoparticle-based targeting of vaccine compounds to skin antigen-presenting cells by hair follicles and their transport in mice. J Invest Dermatol 2009;129:1156-1164.

9 Vogt A, Combadiere B, Hadam S, Stieler KM, Lademann J, Schaefer H, Autran B, Sterry W, Blume-Peytavi U: $40 \mathrm{~nm}$, but not 750 or 1,500 $\mathrm{nm}$, nanoparticles enter epidermal CDla+ cells after transcutaneous application on human skin. J Invest Dermatol 2006;126:13161322.

10 Vogt A, Hadam S, Deckert I, Schmidt J, Stroux A, Afraz Z, Rancan F, Lademann J, Combadiere B, Blume-Peytavi U: Hair follicle targeting, penetration enhancement and Langerhans cell activation make cyanoacrylate skin surface stripping a promising delivery technique for transcutaneous immunization with large molecules and particle-based vaccines. Exp Dermatol 2014;24:73-75.

11 Perkins MA, Osterhues MA, Farage MA, Robinson MK: A noninvasive method to assess skin irritation and compromised skin conditions using simple tape adsorption of molecular markers of inflammation. Skin Res Technol 2001;7:227-237.

12 Clausen ML, Slotved HC, Krogfelt KA, Agner $\mathrm{T}$ : Tape stripping technique for stratum corneum protein analysis. Sci Rep 2016;6:19918.

13 Doge N, Avetisyan A, Hadam S, Pfannes EK, Rancan F, Blume-Peytavi U, Vogt A: Assessment of skin barrier function and biochemical changes of ex vivo human skin in response to physical and chemical barrier disruption. Eur J Pharm Biopharm 2017;116:138-148.
14 Marttin E, Neelissen-Subnel MTA, De Haan FHN, Boddé HE: A critical comparison of methods to quantify stratum corneum removed by tape stripping. Skin Pharmacol Physiol 1996;9:69-77.

15 Wood LC, Stalder AK, Liou A, Campbell IL, Grunfeld C, Elias PM, Feingold KR: Barrier disruption increases gene expression of cytokines and the $55 \mathrm{kD}$ TNF receptor in murine skin. Exp Dermatol 1997;6:98-104.

16 Nickoloff BJ, Naidu Y: Perturbation of epidermal barrier function correlates with initiation of cytokine cascade in human skin. J Am Acad Dermatol 1994;30:535-546.

17 Ahrens K, Schunck M, Podda GF, Meingassner J, Stuetz A, Schroder JM, Harder J, Proksch E: Mechanical and metabolic injury to the skin barrier leads to increased expression of murine beta-defensin-1, -3 , and -14 . J Invest Dermatol 2011;131:443-452.

18 Dickel H, Gambichler T, Kamphowe J, Altmeyer P, Skrygan M: Standardized tape stripping prior to patch testing induces upregulation of Hsp90, Hsp70, IL-33, TNF-alpha, and IL-8/CXCL8 mRNA: new insights into the involvement of "alarmins." Contact Dermatitis 2010;63:215-222.

19 Combadiere B, Vogt A, Mahe B, Costagliola D, Hadam S, Bonduelle O, Sterry W, Staszewski S, Schaefer H, van der Werf S, Katlama C, Autran B, Blume-Peytavi U: Preferential amplification of CD8 effector-T cells after transcutaneous application of an inactivated influenza vaccine: a randomized phase I trial. PLoS One 2010;5:e10818.

20 Vogt A, Hadam S, Pfannes EB, Blume-Peytavi $U$, Combadiere $B$ : Ex vivo and in vivo studies support safety and non-invasiveness of hair follicle targeting for transcutaneous immunization strategies. 45th Annu ESDR Meet, Rotterdam, 2015. 
21 Loffler H, Dreher F, Maibach HI: Stratum corneum adhesive tape stripping: influence of anatomical site, application pressure, duration and removal. Br J Dermatol 2004;151: 746-752.

22 Breternitz M, Flach M, Prassler J, Elsner P, Fluhr JW: Acute barrier disruption by adhesive tapes is influenced by pressure, time and anatomical location: integrity and cohesion assessed by sequential tape stripping. A randomized, controlled study. Br J Dermatol 2007;156:231-240.

23 Combadiere B, Liard C: Transcutaneous and intradermal vaccination. Hum Vaccin 2011;7: 811-827.

24 Kupper TS, Fuhlbrigge RC: Immune surveillance in the skin: mechanisms and clinical consequences. Nat Rev Immunol 2004;4:211222.

25 Nicolas JF, Guy B: Intradermal, epidermal and transcutaneous vaccination: from immunology to clinical practice. Expert Rev Vaccines 2008;7:1201-1214.

26 Dinarello CA: Immunological and inflammatory functions of the interleukin-1 family. Annu Rev Immunol 2009;27:519-550.

27 De Jongh CM, Verberk MM, Spiekstra SW, Gibbs S, Kezic S: Cytokines at different stratum corneum levels in normal and sodium lauryl sulphate-irritated skin. Skin Res Technol 2007;13:390-398.

28 Wood LC, Elias PM, Calhoun C, Tsai JC, Grunfeld C, Feingold KR: Barrier disruption stimulates interleukin-1 alpha expression and release from a pre-formed pool in murine epidermis. J Invest Dermatol 1996;106:397-403.

29 Karande P, Mitragotri S: Transcutaneous immunization: an overview of advantages, disease targets, vaccines, and delivery technologies. Annu Rev Chem Biomol Eng 2010;1: 175-201.
30 Vogt A, Mahe B, Costagliola D, Bonduelle O, Hadam S, Schaefer G, Schaefer H, Katlama C, Sterry W, Autran B, Blume-Peytavi U, Combadiere B: Transcutaneous anti-influenza vaccination promotes both $\mathrm{CD} 4$ and $\mathrm{CD} 8 \mathrm{~T}$ cell immune responses in humans. J Immunol 2008; 180:1482-1489.

31 Wang XP, Schunck M, Kallen KJ, Neumann C, Trautwein C, Rose-John S, Proksch E: The interleukin- 6 cytokine system regulates epidermal permeability barrier homeostasis. J Invest Dermatol 2004;123:124-131.

32 Amarbayasgalan T, Takahashi H, Dekio I, Morita E: Interleukin-8 content in the stratum corneum as an indicator of the severity of inflammation in the lesions of atopic dermatitis. Int Arch All Immunol 2013;160:63-74.

33 Schroder JM, Mrowietz U, Morita E, Christophers E: Purification and partial biochemical characterization of a human monocyte-derived, neutrophil-activating peptide that lacks interleukin 1 activity. J Immunol 1987;139: 3474-3483.

34 Duffy D, Perrin H, Abadie V, Benhabiles N, Boissonnas A, Liard C, Descours B, Reboulleau D, Bonduelle O, Verrier B, Van Rooijen $\mathrm{N}$, Combadière $\mathrm{C}$, Combadière B: Neutrophils transport antigen from the dermis to the bone marrow, initiating a source of memory CD8+ T cells. Immunity 2012;37:917-929.

35 Lee HY, Stieger M, Yawalkar N, Kakeda M: Cytokines and chemokines in irritant contact dermatitis. Mediat Inflamm 2013;2013: 916497.
36 Hickman HD, Reynoso GV, Ngudiankama BF, Cush SS, Gibbs J, Bennink JR, Yewdell JW: CXCR3 chemokine receptor enables local CD8(+) T cell migration for the destruction of virus-infected cells. Immunity 2015;42:524537.

37 Dickson MC, Ludbrook VJ, Perry HC, Wilson PA, Garthside SJ, Binks MH: A model of skin inflammation in humans leads to a rapid and reproducible increase in the interferon response signature: a potential translational model for drug development. Inflamm Res 2015;64:171-183.

38 Onoue A, Kabashima K, Kobayashi M, Mori $\mathrm{T}$, Tokura Y: Induction of eosinophil- and Th2-attracting epidermal chemokines and cutaneous late-phase reaction in tapestripped skin. Exp Dermatol 2009;18:10361043.

39 Koria P, Brazeau D, Kirkwood K, Hayden P, Klausner M, Andreadis ST: Gene expression profile of tissue engineered skin subjected to acute barrier disruption. J Invest Dermatol 2003;121:368-382.

40 Mittal A, Schulze K, Ebensen T, Weissmann S, Hansen S, Lehr CM, Guzman CA: Efficient nanoparticle-mediated needle-free transcutaneous vaccination via hair follicles requires adjuvantation. Nanomed Nanotechnol Biol Med 2015;11:147-154

41 Li N, Peng LH, Chen X, Nakagawa S, Gao JQ: Effective transcutaneous immunization by antigen-loaded flexible liposome in vivo. Int $\mathrm{J}$ Nanomed 2011;6:3241-3250.

42 Mittal A, Raber AS, Schaefer UF, Weissmann S, Ebensen T, Schulze K, Guzman CA, Lehr CM, Hansen S: Non-invasive delivery of nanoparticles to hair follicles: a perspective for transcutaneous immunization. Vaccine 2013;31:3442-3451. 